\title{
Light Refraction and Reflection near a Black Hole
}

\author{
Young Hwan Yun, Kiho Jang \\ Zero Theoretical Physics Laboratory, Seoul, Republic of Korea \\ Email: yhyun690@naver.com
}

How to cite this paper: Yun, Y.H. and Jang, K. (2022) Light Refraction and Reflection near a Black Hole. Journal of High Energy Physics, Gravitation and Cosmology, 8, 228-236.

https://doi.org/10.4236/jhepgc.2022.81016

Received: December 10, 2021

Accepted: January 21, 2022

Published: January 24, 2022

Copyright (c) 2022 by author(s) and Scientific Research Publishing Inc. This work is licensed under the Creative Commons Attribution International License (CC BY 4.0).

http://creativecommons.org/licenses/by/4.0/

\begin{abstract}
In this paper, we have calculated the angle of refraction that light travels approaching to the strong gravitational field like a black hole by combining the general relativity and the classical Snell's law, assuming that the gravitational field can act as a non-vacuum filled with medium of some coefficients. We have found that the value of refracted angle exactly coincides with the value from the Einstein's relativity theory in a weak gravitational field. From this optical interpretation of the traveling of light near a black hole, we have suggested that there might have the reflection phenomenon and investigated that the total reflection occurs at the surface of a black hole. Regardless this might cause controversy, we can explain the recent observation that light reflects from a black hole.
\end{abstract}

\section{Keywords}

Black Hole, Snell's Law, Relativity, Refraction, Reflection

\section{Introduction}

According to the general relativity theory, the path of light bends by gravity, and the speed of light decreases as it approaches the surface of gravitation, and finally light stops at the Schwarzschild radius (i.e. $\mathrm{r}=2 \mathrm{GM}$ ). A relativistic interpretation says that space-time is curved in the gravitational field. In curved spacetime, light also bends along the curved space-time and proceeds. In this paper, we try to relate this phenomenon to classical interpretation. Snell's law relates the ratio of the speed of light to the angle of refraction of the medium within the light travels. Considering that gravity causes the speed difference of light to change the medium, Snell's law according to the speed difference can be applied to calculate the angle of refraction. If this calculation of the refraction angle is 
the same as the angle obtained from the relativity theory, we can regard this logic as reasonable. If the refraction analysis through Snell's law is valid, we can't help but consider reflection, so we'll cover this as well.

\section{Relativistic Optics near a Black Hole}

In this chapter, the speed of light and its degradation due to gravity are used in Snell's law, and we can calculate the bending angle quantitatively.

In Figure 1, when we consider Snell's law first, the effective refraction ratio in the general medium can be described as Equation (2-1) and Equation (2-2), where $v_{1}$, and $v_{2}$ are the speed of light in the media, $c$ is the speed of light in vacuum, and $n$ is refraction ratio.

$$
\begin{aligned}
& \text { Absolute refraction ratio }=\frac{c}{v} \\
& \text { Relative refraction ratio }=\frac{v_{1}}{v_{2}}
\end{aligned}
$$

In order to know the velocity of light in the gravitational field, we use the equation of the general relativity. In this case, we only discuss non-rotating spherical objects and we can use the Schwarzschild solution [1].

At the spherical coordinate system, space-time interval, using Schwarzschild metric, is Equation (2-3) [1]

$$
\mathrm{d} s^{2}=g_{\mu v} \mathrm{~d} x^{\mu} \mathrm{d} x^{v}=\left(1-\frac{2 G M}{r}\right) \mathrm{d} t^{2}-\frac{\mathrm{d} r^{2}}{1-\frac{2 G M}{r}}-r^{2} \mathrm{~d} \theta^{2}-r^{2} \sin ^{2} \theta \mathrm{d} \phi^{2}
$$

where $G$ is gravitational constant and $M$ is mass of planet.

In case of light, Equation (2-3) becomes Equation (2-4), and Equation (2-5) is derived.

$$
\begin{gathered}
0=\mathrm{d} s^{2}=g_{\mu \nu} \mathrm{d} x^{\mu} \mathrm{d} x^{v} \\
v=\frac{\mathrm{d} r}{\mathrm{~d} t}=1-\frac{2 G M}{r}
\end{gathered}
$$

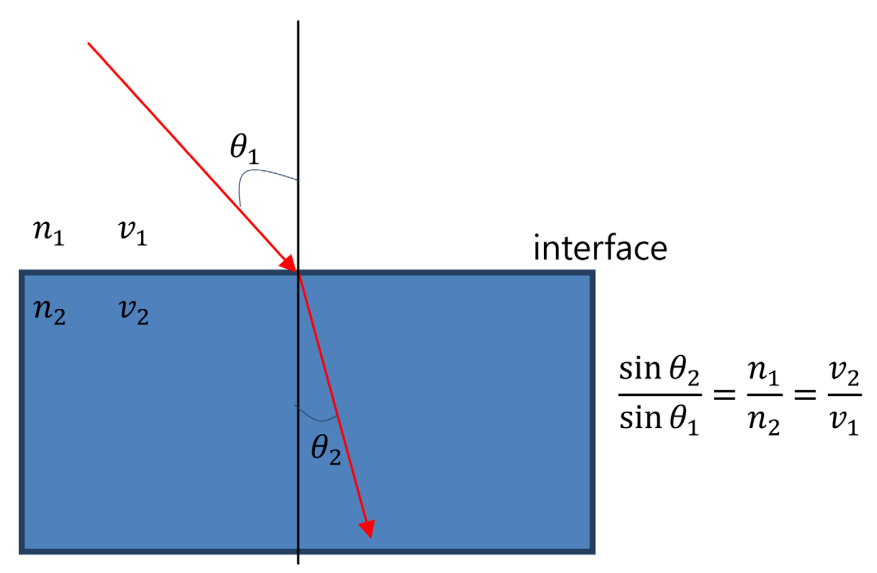

Figure 1. Snell's law. 
The relative refraction ratio in the gravitational field can be defined using Equation (2-2) and Equation (2-5).

$$
\frac{n_{2}}{n_{1}}(\text { effective ratio })=\frac{1-\frac{2 G M}{r_{1}}}{1-\frac{2 G M}{r_{2}}}
$$

If $r_{1} \gg 2 G M$, in free space, we can set the $2 G M / r_{1} \approx 0$.

In this case, with the help of Equation (2-6), we can make Equation (2-7).

$$
\frac{n_{2}}{n_{1}}=\frac{\sin \theta_{1}}{\sin \theta_{2}}=\frac{V_{1}}{V_{2}}=\frac{C}{V} \approx \frac{1}{1-2 G M / r_{2}}
$$

In case of spherical coordinate and a multi-layers system like Figure 2, we can assume the layered refracted angles like Equation (2-8).

$$
\theta_{2} \neq \theta_{3}, \theta_{4} \neq \theta_{5}, \cdots, \theta_{2 k-1} \neq \theta_{2 k}
$$

And we can obtain the relations from Equation (2-9) to Equation (2-12).

$$
\begin{gathered}
\frac{n_{2}}{n_{1}}=\frac{\sin \theta_{1}}{\sin \theta_{2}} \\
\frac{n_{4}}{n_{3}}=\frac{\sin \theta_{3}}{\sin \theta_{4}} \\
\frac{n_{6}}{n_{5}}=\frac{\sin \theta_{5}}{\sin \theta_{6}}
\end{gathered}
$$

$$
\frac{n_{k}}{n_{k-1}}=\frac{\sin \theta_{k-1}}{\sin \theta_{k}}
$$

To find the relationship between the outermost Equation (2-9) and the innermost Equation (2-12), we can multiply each other, and we have Equation $(2-13)$

$$
\frac{n_{k}}{n_{k-1}} \frac{n_{2}}{n_{1}}=\frac{\sin \theta_{k-1}}{\sin \theta_{k}} \frac{\sin \theta_{1}}{\sin \theta_{2}}
$$

where

$$
\alpha \equiv \frac{n_{k-1}}{n_{2}} \frac{\sin \theta_{k-1}}{\sin \theta_{2}}
$$

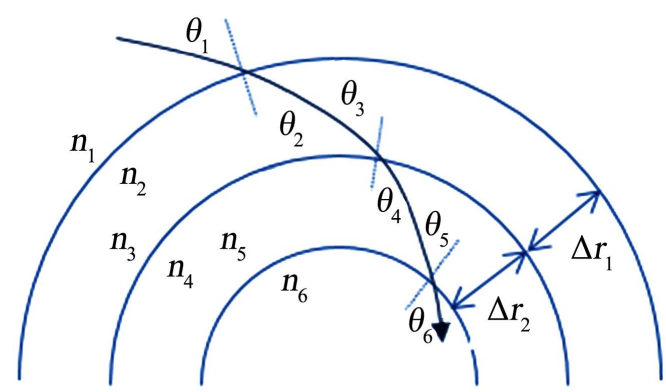

Figure 2. Light refraction by gravitational field. 
Therefore, the relationship between the outermost and the innermost is a modified Snell's law as Equation (2-15).

$$
\frac{n_{k}}{n_{1}}=\frac{\sin \theta_{1}}{\sin \theta_{k}} \alpha
$$

Using this formula, the modified Snell's law can be applied from the outermost free space to the space affected by gravity through the medium coefficient. Using Equation (2-7), it can be made as Equation (2-16).

$$
\frac{n_{k}}{n_{1}} \approx \frac{1}{1-2 G M / r_{k}} \alpha
$$

\section{Refracted Angle in a Weak Gravitational Field}

In this chapter, we would like to calculate the angle of refraction quantitatively. This calculation is already done by the general relativity theory in the weak gravitational field and we also start from the weak field. To know the refracted angle in a weak gravitational field, we can only consider a two-layer system with coefficients $n_{1}$ and $n_{2}$. Since it does not enter deep into the gravitational field, we can have $\alpha=1$ in Equation (2-15). Now we describe the equations using $\Delta \theta$ like Equation (3-1).

$$
\Delta \theta_{1}=\theta_{1}-\theta_{2}
$$

Using Equation (3-1) we can express $\sin \theta_{1}$ in terms of $\Delta \theta_{1}$,

$$
\begin{aligned}
\sin \theta_{1} & =\sin \left(\theta_{2}+\Delta \theta_{1}\right) \\
& =\sin \theta_{2} \cos \Delta \theta_{1}+\sin \Delta \theta_{1} \cos \theta_{2}
\end{aligned}
$$

If we assume that $\Delta \theta_{1}$ is very small, we have this

$$
\sin \theta_{1} \approx \sin \theta_{2}+\Delta \theta_{1} \cos \theta_{2}
$$

By using Equation (3-4), we can change the numerator of Equation (2-9) like Equation (3.5) and Equation (3-6).

$$
\begin{aligned}
\frac{n_{2}}{n_{1}} & \approx \frac{\sin \theta_{2}+\Delta \theta_{1} \cos \theta_{2}}{\sin \theta_{2}} \\
& =1+\Delta \theta_{1} \cot \theta_{2}
\end{aligned}
$$

We can also change the denominator of Equation (2-9). Before doing that, we can change $\sin \theta_{2}$ to this approximation.

$$
\begin{aligned}
\sin \theta_{2} & =\sin \left(\theta_{1}-\Delta \theta_{1}\right) \\
& =\sin \theta_{1} \cos \Delta \theta_{1}-\sin \Delta \theta_{1} \cos \theta_{1} \\
& \approx \sin \theta_{1}-\Delta \theta_{1} \cos \theta_{1}
\end{aligned}
$$

Finally, we obtain an effective refraction ratio like Equation (3-10) and Equation (3-11).

$$
\frac{n_{2}}{n_{1}} \approx \frac{\sin \theta_{1}}{\sin \theta_{1}-\Delta \theta_{1} \cos \theta_{1}}
$$




$$
=\frac{1}{1-\Delta \theta_{1} \cot \theta_{1}}
$$

If we combine Equation (3-6) and Equation (2-9), we have Equation (3-12).

$$
\frac{n_{2}}{n_{1}}=\frac{\sin \theta_{1}}{\sin \theta_{2}} \approx 1+\Delta \theta_{1} \cot \theta_{2}
$$

If we combine Equation (3-11) and Equation (2-9), we have Equation (3-13).

$$
\frac{n_{2}}{n_{1}}=\frac{\sin \theta_{1}}{\sin \theta_{2}} \approx \frac{1}{1-\Delta \theta_{1} \cot \theta_{1}}
$$

By using Equation (3-12) and Equation (2-7), we have Equation (3-14).

$$
1+\Delta \theta_{1} \cot \theta_{2} \approx \frac{1}{1-2 G M / r}
$$

Rearranging Equation (3-14) for $\Delta \theta_{1}$, we have Equation (3-15).

$$
\Delta \theta_{1} \approx \frac{2 G M / r}{1-2 G M / r} \tan \theta_{2}
$$

Using Equation (3-13) and Equation (2-7), we have Equation (3-16).

$$
\frac{1}{1-\Delta \theta_{1} \cot \theta_{1}} \approx \frac{1}{1-2 G M / r}
$$

Rearranging Equation (3-16) for $\Delta \theta_{1}$, we have Equation (3-17).

$$
\Delta \theta_{1} \approx \frac{2 G M}{r} \tan \theta_{1}
$$

From Equation (3-15) and Equation (3-17), we have Equation (3-18).

$$
\Delta \theta_{1} \approx \frac{2 G M / r}{1-2 G M / r} \tan \theta_{2} \approx \frac{2 G M}{r} \tan \theta_{1}
$$

Rearranging Equation (3-18), we have Equation (3-19).

$$
\frac{1 / \tan \theta_{2}}{1 / \tan \theta_{1}}=\frac{\tan \theta_{1}}{\tan \theta_{2}} \approx \frac{1}{1-2 G M / r}
$$

When the light is deflected toward the center of gravity like Figure 2, we may have $\theta_{1}>\theta_{2}$.

From Equation (3-19) and Equation (2-7), we can assume mathematically two cases.

The first case is Equation (3-20).

$$
\frac{\sin \theta_{1}}{\sin \theta_{2}} \approx \frac{\tan \theta_{1}}{\tan \theta_{2}}
$$

And the other case is Equation (3-21).

$$
\frac{\sin \theta_{1}}{\sin \theta_{2}} \approx \frac{1 / \tan \theta_{2}}{1 / \tan \theta_{1}}
$$

Equation (3-20) is the case where $\sin \theta_{1} \approx \tan \theta_{1}$ and $\sin \theta_{2} \approx \tan \theta_{2}$ and it means $\theta_{1} \approx \theta_{2} \approx 0$, which is inconsistent with the case of Figure 3. Thus we consider Equation (3-21) case only. 


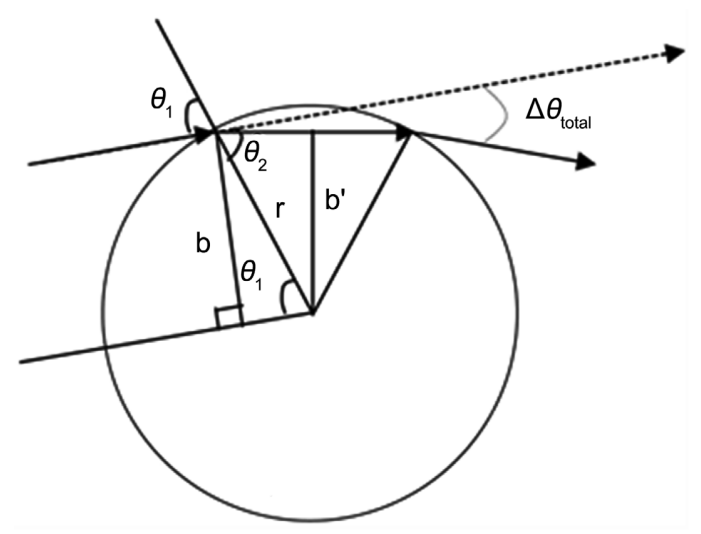

Figure 3. A slightly refracted light path at the boundary of different matter.

From Equation (3-21), we have Equation (3-22) and Equation (3-23).

$$
\begin{aligned}
& \sin \theta_{2} \approx \frac{1}{\tan \theta_{1}} \\
& \sin \theta_{1} \approx \frac{1}{\tan \theta_{2}}
\end{aligned}
$$

Using Equation (3-18) and Equation (3-22), we have Equation (3-24).

$$
\Delta \theta_{1} \approx \frac{2 G M}{r} \tan \theta_{1} \approx \frac{2 G M}{r} \frac{1}{\sin \theta_{2}}
$$

In Figure 3, we can know $r \sin \theta_{2}=b^{\prime}$, we have Equation (3-25).

$$
\Delta \theta_{1} \approx \frac{2 G M}{b^{\prime}}
$$

As there are two symmetric refracted angles in Figure 3, so the total refracted angle is Equation (3-26).

$$
\therefore \Delta \theta_{\text {total }} \approx 2 \frac{2 G M}{b^{\prime}}=\frac{4 G M}{b^{\prime}}
$$

It is noticeable that Equation (3-26) is same as the angle calculated by Einstein [2] [3]. This result is not a coincidence, but a reasonable result of the assumption of the effective medium caused by the relativistic field distribution of gravity.

\section{Reflection on a Black Hole}

The deflected angle of light in the gravitational field is calculated in the previous chapter with the help of the optical property of light refraction in the medium. If the light is refracted, it naturally implies that there is also reflection. Thus the reflection and transmission coefficients are investigated in this chapter (Figure 4).

There are two cases of transmission coefficients, TE mode, and TM mode [4]. Electric fields ratio perpendicular to plane of incidence is Equation (4-1).

$$
\frac{E_{1}^{\prime}}{E_{1}}=\frac{2 n_{1} \cos \theta_{1}}{n_{1} \cos \theta_{1}+\frac{\mu}{\mu^{\prime}} \sqrt{n_{2}^{2}-n_{1}^{2} \sin ^{2} \theta_{1}}}
$$



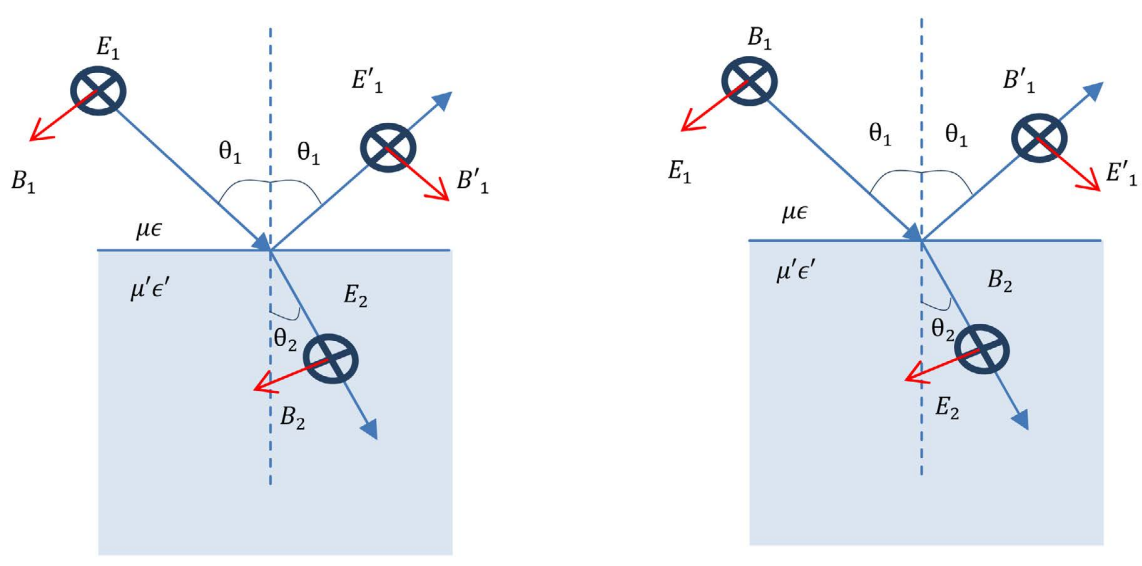

Figure 4. left: s-polarization (TE mode), right: p-polarization (TM mode).

$$
\frac{E_{2}}{E_{1}}=\frac{n_{1} \cos \theta_{1}-\frac{\mu}{\mu^{\prime}} \sqrt{n_{2}^{2}-n_{1}^{2} \sin ^{2} \theta_{1}}}{n_{1} \cos \theta_{1}+\frac{\mu}{\mu^{\prime}} \sqrt{n_{2}^{2}-n_{1}^{2} \sin ^{2} \theta_{1}}}
$$

Electric fields ratio parallel to plane of incidence is Equation (4-2).

$$
\begin{aligned}
& \frac{E_{1}^{\prime}}{E_{1}}=\frac{2 n_{1} n_{2} \cos \theta_{1}}{\frac{\mu}{\mu^{\prime}} n_{2}^{2} \cos \theta_{1}+n_{1} \sqrt{n_{2}^{2}-n_{1}^{2} \sin ^{2} \theta_{1}}} \\
& \frac{E_{2}}{E_{1}}=\frac{\frac{\mu}{\mu^{\prime}} n_{2}^{2} \cos \theta_{1}-n_{1} \sqrt{n_{2}^{2}-n_{1}^{2} \sin ^{2} \theta_{1}}}{\frac{\mu}{\mu^{\prime}} n_{2}^{2} \cos \theta_{1}+n_{1} \sqrt{n_{2}^{2}-n_{1}^{2} \sin ^{2} \theta_{1}}}
\end{aligned}
$$

For normal incidence, both Equations (4-1) and (4-2) reduce to Equation (4-3).

$$
\begin{gathered}
\frac{E_{1}^{\prime}}{E_{1}}=\frac{2 n_{1}}{n_{1}+n_{2}}=\frac{2}{1+\frac{n_{2}}{n_{1}}} \\
\frac{E_{2}}{E_{1}}= \pm \frac{n_{2}-n_{1}}{n_{2}+n_{1}}= \pm \frac{\frac{n_{2}}{n_{1}}-1}{\frac{n_{2}}{n_{1}}+1}
\end{gathered}
$$

Here, if we look at the case of light entering the gravitational field from the outermost shell, we can use the modified Snell's law like Equation (4-4).

$$
\frac{E_{1}^{\prime}}{E_{1}}=\frac{2}{1+\frac{n_{k}}{n_{1}} \alpha}
$$

And the reflection coefficients for both TE and TM is Equation (4-5) [4].

$$
\frac{E_{2}}{E_{1}}=\frac{\frac{n_{k}}{n_{1}} \alpha-1}{\frac{n_{k}}{n_{1}} \alpha+1}
$$


According to Equation (2-16), When $r_{2} \rightarrow 2 G M$, the refraction ratio becomes infinite (that is, $\frac{n_{k}}{n_{1}} \rightarrow \infty$ ). With these conditions, the transmission coefficients go to 0 and the reflection coefficients go to 1. In Equation (2-15), if $n_{1}$ and $n_{2}$ are in contact, $\alpha=1$. But if they are apart, $\alpha$ has a certain finite value. In any case it holds that $\frac{n_{2}}{n_{1}} \rightarrow \infty$, and it becomes Equation (4-6) and Equation $(4-7)$.

$$
\begin{aligned}
& \left|\frac{E_{1}^{\prime}}{E_{1}}\right| \rightarrow 0 \\
& \left|\frac{E_{2}}{E_{1}}\right| \rightarrow 1
\end{aligned}
$$

Magnetic fields also have the same properties as Equation (4-6) and Equation (4-7). From Equation (4-6) and Equation (4-7), it means that there happens total reflection at Schwarzschild radius. Einstein calculated the bend angle using the geodesic equation. But there was no light reflection in his calculation. In our approach, we suggest there happens light reflection near a black hole, but the reflection is not a local phenomenon. If we interpret qualitatively the meaning so far, light is not reflected all at once. Instead of it, as light travels from the outermost free space to the surface of a black hole, a bunch of light is reflected more and more, and at a certain point in a distance, the amount of reflection decreases. There is no light that can reach the surface of a black hole finally. This is diagrammed in Figure 5.

Although the approach is different, other studies also have similar claims [5] [6] [7] [8]. Most of them are based on quantum mechanical logic, but our approach is relativistic optical logic. It is interesting to note that the goals of both approaches expecting the reflection near a black hole are same. There is also observed data supporting the total reflection phenomenon [9]. According to this report, galaxy Sagittarius has a tendency to blow away $99.99 \%$ of the matter available for its consumption. This is an observational fact, but a theoretical basis is still required. As one of an interpretation of this, it is believed that the absorbed materials are spit out again due to the collision energy between them. We also suggest that the explanation developed in this paper may contribute in part.

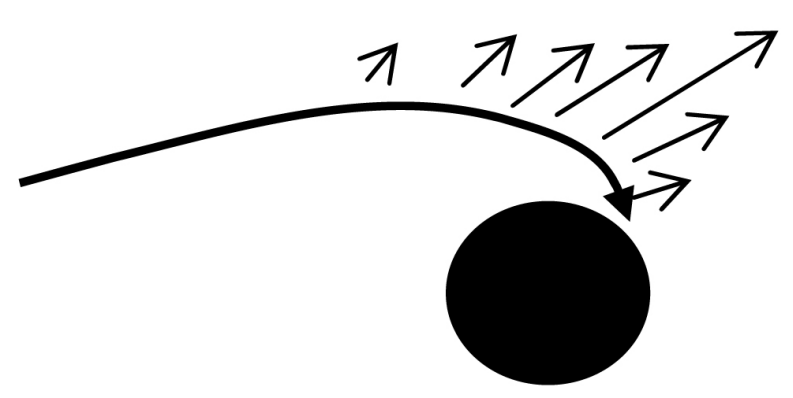

Figure 5. Reflected lights when a group of photons is approaching a black hole. 


\section{Conclusion}

We have applied the change of the speed of light in a gravitational field to the classical interpretation that the difference in speed of light mentioned in Snell's law is directly related to the angle of refraction, and have found the refracted angle of light. The result is the same as previously calculated by Einstein. This confirms that the combination of relativity and classical interpretation is also meaningful. However, where there is refraction, there is also a reflection phenomenon. As a result of calculating this reflection, when light approaches the surface of a black hole, it is reflected gradually, leaving nothing that reaches the surface of a black hole finally. Although this is a phenomenon that does not exist in the conventional relativity theory, it is interesting that other arguments have interpreted from the quantum theoretical point of view and have the same result. Additional study is also needed to find the same interpretation in the inertial coordinate system in order to have a more robust explanation.

\section{Conflicts of Interest}

The authors declare no conflicts of interest regarding the publication of this paper.

\section{References}

[1] Buchdahl, H.A. (1985) Isotropic Coordinates and Schwarzschild Metric. International Journal of Theoretical Physics, 24, 731-739. https://doi.org/10.1007/BF00670880

[2] Ohanian, H.C. and Ruffini, R. (1994) Gravitation and Spacetime. 2nd Edition, W. W. Norton \& Company, New York.

[3] Wald, R.M. (1984) General Relativity. University of Chicago Press, Chicago, 144146.

[4] Jackson, J.D. (1998) Classical Electrodynamics. Wiley, Hoboken, 278-282.

[5] Kuchiev, M.Yu. (2004) Reflection on Event Horizon for Collapsing Black Holes. arXiv:0411009v1[gr-qc]

[6] Kuchiev, M.Yu. (2003) Reflection from Black Holes. arXiv:0310008v1[gr-qc]

[7] Good, M.R.R., Anderson, P.R. and Evans, C.R. (2016) Mirror Reflections of a Black Hole. Physical Review D, 94, Article ID: 065010.

[8] Good, M.R.R. (2016) Mirror Reflections on a Black Hole. arXiv: 1602.00683v2[gr-qc]

[9] Shcherbakov, R. (2010) Supermassive Black Hole Struggles to Swallow Milky Way. 215th Annual Meeting of the American Astronomical Society (AAS). https://physicsworld.com/a/supermassive-black-hole-struggles-to-swallow-milky-w ay/ 